\title{
Импликативные расширения регулярных логик Клини
}

H. E. TOMOBA

\begin{abstract}
The family of regular 3-valued Kleene's logics (strong, weak and intermediate) are considered as bases for other logics. Extentions of regular logics by implicative connectives ("natural" implication) are considered. In conclusion all exententions presented as a lattice.
\end{abstract}

Ключевые слова: трехзначные логики, регулярные логики Клини, импликация, расширения регулярных логик

\section{1 Введение}

В [3] С. Клини описал свойство регулярности, оно является необходимым условием для того, чтобы логические связки моделировали рекурсивные функции, вычисление значения которых никогда не оканчивается. С этой целью, пишет Клини, таблицы для связок должны быть регулярными в следующем смысле: «данный столбеи, (строка) содержит 1 в строке (столбце) для 1/2 только при условии, что этот столбеи, (строка) состоит целиком из 1; аналогично для 0». В этой же работе Клини представил две трехзначные регулярные логики - сильную логику Клини $\mathbf{K}_{\mathbf{3}}$ и слабую логику Клини $\mathbf{K}_{\mathbf{3}}^{\mathbf{w}}$. В дальнейшем, следуя С. Клини $[3, \S 64]$, под трехзначной логикой будет пониматься некоторое множество логических связок.

М. Фиттинг [14] указал на существование промежуточной регулярной логики Lisp $\left(\mathbf{K}_{\mathbf{3}}\right)$. Логика Lisp, а также взаимоотношения между регулярными логиками подробно рассмотрены в $[4]^{1}$.

\footnotetext{
${ }^{1} \mathrm{~B}$ этой же работе представлена еще одна промежуточная регулярная логика Twin Lisp, которая функционально эквивалентна Lisp. Поскольку данные системы функционально эквивалентны, достаточно рассмотреть одну.
} 
Итак, в качестве регулярных логик будем рассматривать логики вида: $\{\sim, \vee, \wedge\}$, где $\sim-$ регулярное отрицание, $\vee, \wedge-$ регулярные дизъюнкция и конъюнкция соответственно ${ }^{2}$.

Сильная логика Клини $\mathbf{K}_{\mathbf{3}}$ есть логика с исходными связками $\sim^{3}, \vee, \wedge$, где $\vee, \wedge$ определяются сильными регулярными таблицами:

\begin{tabular}{|c|ccc|}
\hline$\vee$ & 1 & $\frac{1}{2}$ & 0 \\
\hline 1 & 1 & 1 & 1 \\
$\frac{1}{2}$ & 1 & $\frac{1}{2}$ & $\frac{1}{2}$ \\
0 & 1 & $\frac{1}{2}$ & 0 \\
\hline
\end{tabular}

\begin{tabular}{|c|ccc|}
\hline$\wedge$ & 1 & $\frac{1}{2}$ & 0 \\
\hline 1 & 1 & $\frac{1}{2}$ & 0 \\
$\frac{1}{2}$ & $\frac{1}{2}$ & $\frac{1}{2}$ & 0 \\
0 & 0 & 0 & 0 \\
\hline
\end{tabular}

Слабая логика Клини $\mathbf{K}_{3}^{\mathbf{w}}$ есть логика с исходными связками $\sim, \cup, \cap$, где $\cup, \cap$ определяются слабыми регулярными таблицами:

\begin{tabular}{|c|ccc|}
\hline$\cup$ & 1 & $\frac{1}{2}$ & 0 \\
\hline 1 & 1 & $\frac{1}{2}$ & 1 \\
$\frac{1}{2}$ & $\frac{1}{2}$ & $\frac{1}{2}$ & $\frac{1}{2}$ \\
0 & 1 & $\frac{1}{2}$ & 0 \\
\hline
\end{tabular}

\begin{tabular}{|c|ccc|}
\hline$\cap$ & 1 & $\frac{1}{2}$ & 0 \\
\hline 1 & 1 & $\frac{1}{2}$ & 0 \\
$\frac{1}{2}$ & $\frac{1}{2}$ & $\frac{1}{2}$ & $\frac{1}{2}$ \\
0 & 0 & $\frac{1}{2}$ & 0 \\
\hline
\end{tabular}

Промежуточная логика $\mathbf{K}_{\mathbf{3}}$ есть логика с исходными связками $\sim, \vee^{\rightarrow}, \wedge \rightarrow$, где $\vee^{\rightarrow}, \wedge^{\rightarrow}$ определяются регулярными таблицами вида:

\begin{tabular}{|c|ccc|}
\hline$\vee^{\prime}$ & 1 & $\frac{1}{2}$ & 0 \\
\hline 1 & 1 & 1 & 1 \\
$\frac{1}{2}$ & $\frac{1}{2}$ & $\frac{1}{2}$ & $\frac{1}{2}$ \\
0 & 1 & $\frac{1}{2}$ & 0 \\
\hline
\end{tabular}

\begin{tabular}{|c|ccc|}
\hline$\wedge^{\rightarrow}$ & 1 & $\frac{1}{2}$ & 0 \\
\hline 1 & 1 & $\frac{1}{2}$ & 0 \\
$\frac{1}{2}$ & $\frac{1}{2}$ & $\frac{1}{2}$ & $\frac{1}{2}$ \\
0 & 0 & 0 & 0 \\
\hline
\end{tabular}

Обратим внимание на взаимоотношение между регулярными логиками. В работе [8, с. 425] В.К. Финн определил слабые связки Клини через сильные:

\footnotetext{
2 Заметим, что во всех регулярных логиках Клини в качестве исходных связок достаточно взять отрицание и дизъюнкцию (или конъюнкцию), поскольку все остальные связки классически выразимы через указанные. Однако для дальнейшего удобства в качестве исходных в каждой из регулярных логик были взяты три связки: отрицание, дизъюнкция и конъюнкция.

${ }^{3} \sim$ определяется одинаково в каждой из регулярных логик: $\sim 0=1$, $\sim 1=0, \sim \frac{1}{2}=\frac{1}{2}$.
} 


$$
p \cap q=(p \wedge q) \vee(p \wedge \sim p) \vee(q \wedge \sim q) .
$$

Также было показано, что обратное - определение сильных связок посредством слабых - не может быть осуществлено. Таким образом, $\mathbf{K}_{\mathbf{3}}^{\mathbf{w}}$ функционально вложима в $\mathbf{K}_{\mathbf{3}}$, т.е. $\mathbf{K}_{\mathbf{3}}^{\mathbf{w}} \subset \mathbf{K}_{\mathbf{3}}$. Далее, в работе [4, с. 125] доказано, что логика Lisp является промежуточной между сильной и слабой логиками Клини, т.е. $\mathbf{K}_{\mathbf{3}}^{\mathbf{w}} \subset \mathbf{K}_{\mathbf{3}}$ и $\mathbf{K}_{\mathbf{3}} \subset \mathbf{K}_{\mathbf{3}}$. Обратное же не имеет места. Более того, Е.Ю. Комендантской показано, что семейство регулярных логик Клини образует четырехэлементную решетку по отношению функционального вложения [4].

Существенной особенностью всех трехзначных регулярных логик является то, что при одном выделенном значении 1 в этих логиках класс тавтологий пуст. Это очевидно, поскольку все регулярные связки сохраняют значение $\frac{1}{2}$ при значении аргументов $\frac{1}{2}$. Во всех этих системах связка импликации не присутствует в качестве исходной связки, но ее можно ввести: $p \supset q$ есть $\sim p \vee q$. Теперь, если допустить, что в рассматриваемых регулярных логиках не одно, а два выделенных значения, 1 и $\frac{1}{2}$, то класс тавтологий совпадет со множеством общезначимых формул классической логики $\mathbf{C}_{\mathbf{2}}$, но несложно заметить, что правило modus ponens не сохраняет тавтологию.

Однако возникает вопрос, что если регулярные логики Клини расширить за счет добавления связки импликации, обладающей некоторыми «хорошими» свойствами. Например, мы знаем, что трехзначная логика Бочвара $\mathbf{B}_{\mathbf{3}}$ [1] есть слабая логика Клини $\mathbf{K}_{\mathbf{3}}^{\mathbf{w}}$ с импликацией $\rightarrow_{5}{ }^{4}$, а при построении известной паранепротиворечивой логики PCont [5] берется сильная регулярная логика Клини $\mathbf{K}_{\mathbf{3}}$ с импликацией $\rightarrow_{21}{ }^{5}$. Знаменитую трехзначную логику Лукасевича можно представить как $\mathbf{K}_{\mathbf{3}}+\rightarrow_{2}$. Указанные три логики являются наиболее известными представителями семейства трехзначных логик. См. подробно об этом в [2].

Представляет интерес систематически рассмотреть импликативные расширения всех трех регулярных логик Клини и представить эти расширения в виде решетки. Именно это и является целью данной статьи.

\footnotetext{
${ }^{4}$ Импликации с указанными номерами см. ниже.

${ }^{5}$ Указанная импликация встречается впервые в работе [17].
} 


\section{2 Определения и вспомогательные утверждения}

Введем необходимые определения.

ОПРЕДЕЛЕНИЕ 1. Логика $S$ функционально вложима в логику $S^{\prime}$, если все связки логики $S$ могут быть определены посредством связок логики $S^{\prime}$.

ОПРЕДЕЛЕНИЕ 2. Логика $S$ функционально эквивалентна логике $S^{\prime}$, если

(1) логика $S$ функционально вложима в логику $S^{\prime}$ и

(2) логика $S^{\prime}$ функционально вложима в логику $S$.

ОПРЕДЕЛЕНИЕ 3. Пусть $V_{3}$ есть $\left\{0, \frac{1}{2}, 1\right\}$ и $D$ есть множество выделенных значений. Импликацию $\rightarrow$ будем называть естеcmвенной, если она обладает следующими свойствами:

(1) C-расширение, т.е. ограничение $\rightarrow$ на подмножество $\{0,1\}$ множества $V_{3}$ суть обычная классическая связка импликации.

(2) Нормальность в смысле Лукасевича-Тарского [19, p. 134], т.е. если $x \rightarrow y \in D$ и $x \in D$, то $y \in D$.

(3) Согласованность с частичным порядком на $V_{3}$ : если $x \leq y$, то $x \rightarrow y \in D{ }^{6}$

(4) $x \rightarrow y \in V_{3}$, в остальных случаях.

Тогда, согласно определению 3 , при $D=\{1\}$ имеем всего 6 импликаций:

\begin{tabular}{|c|ccc|}
\hline$\rightarrow$ & 1 & $\frac{1}{2}$ & 0 \\
\hline 1 & 1 & $a$ & 0 \\
$\frac{1}{2}$ & 1 & 1 & $b$ \\
0 & 1 & 1 & 1 \\
\hline
\end{tabular}

\footnotetext{
${ }^{6}$ Заметим, что в [21, р. 179-180] Е. Расёва вводит понятие импликативной логики, откуда следует более строгое условие, чем (3), а именно:

$x \leq y$ е.т.е. $x \rightarrow y \in D$ (отношение предпорядка).

Но тогда выпадает, например, импликация $\rightarrow 21$ из логики PCont и, вообще, выпадают логики с хорошими стандартными свойствами (см. ниже).
} 
где $a \in\left\{0, \frac{1}{2}\right\}$ и $b \in\left\{0, \frac{1}{2}, 1\right\}$.

При $D=\left\{1, \frac{1}{2}\right\}$ имеем 24 импликации:

\begin{tabular}{|c|ccc|}
\hline$\rightarrow$ & 1 & $\frac{1}{2}$ & 0 \\
\hline 1 & 1 & $b$ & 0 \\
$\frac{1}{2}$ & $a$ & $a$ & 0 \\
0 & 1 & $a$ & 1 \\
\hline
\end{tabular}

где $a \in\left\{1, \frac{1}{2}\right\}$ и $b \in\left\{0, \frac{1}{2}, 1\right\}$.

Заметим, что 2 пары импликаций ${ }^{7}$ совпадают как при $D=$ $\{1\}$, так и при $D=\left\{1, \frac{1}{2}\right\}$, поэтому имеется всего 28 уникальных импликаций, удовлетворяющих условиям (1)-(4) определения 3.

Для дальнейшего удобства перенумеруем полученные импликации и приведем соответствующие им таблицы истинности:

$D=\{1\}$

\begin{tabular}{|c|ccc|}
\hline$\rightarrow_{1}$ & 1 & $\frac{1}{2}$ & 0 \\
\hline 1 & 1 & $\frac{1}{2}$ & 0 \\
$\frac{1}{2}$ & 1 & 1 & 0 \\
0 & 1 & 1 & 1 \\
\hline
\end{tabular}

\begin{tabular}{|c|ccc|}
\hline$\rightarrow_{2}$ & 1 & $\frac{1}{2}$ & 0 \\
\hline 1 & 1 & $\frac{1}{2}$ & 0 \\
$\frac{1}{2}$ & 1 & 1 & 1 \\
0 & 1 & 1 & 1 \\
\hline
\end{tabular}

\begin{tabular}{|c|ccc|}
\hline$\rightarrow_{3}$ & 1 & $\frac{1}{2}$ & 0 \\
\hline 1 & 1 & $\frac{1}{2}$ & 0 \\
$\frac{1}{2}$ & 1 & 1 & $\frac{1}{2}$ \\
0 & 1 & 1 & 1 \\
\hline
\end{tabular}

\begin{tabular}{|c|ccc|}
\hline$\rightarrow_{4}$ & 1 & $\frac{1}{2}$ & 0 \\
\hline 1 & 1 & 0 & 0 \\
$\frac{1}{2}$ & 1 & 1 & 0 \\
0 & 1 & 1 & 1 \\
\hline
\end{tabular}

\begin{tabular}{|c|ccc|}
\hline$\rightarrow_{5}$ & 1 & $\frac{1}{2}$ & 0 \\
\hline 1 & 1 & 0 & 0 \\
$\frac{1}{2}$ & 1 & 1 & 1 \\
0 & 1 & 1 & 1 \\
\hline
\end{tabular}

\begin{tabular}{|c|ccc|}
\hline$\rightarrow_{6}$ & 1 & $\frac{1}{2}$ & 0 \\
\hline 1 & 1 & 0 & 0 \\
$\frac{1}{2}$ & 1 & 1 & $\frac{1}{2}$ \\
0 & 1 & 1 & 1 \\
\hline
\end{tabular}

$D=\left\{1, \frac{1}{2}\right\}$

\begin{tabular}{|c|ccc|}
\hline$\rightarrow_{7}$ & 1 & $\frac{1}{2}$ & 0 \\
\hline 1 & 1 & 1 & 0 \\
$\frac{1}{2}$ & 1 & 1 & 0 \\
0 & 1 & 1 & 1 \\
\hline
\end{tabular}

\begin{tabular}{|c|ccc|}
\hline$\rightarrow_{8}$ & 1 & $\frac{1}{2}$ & 0 \\
\hline 1 & 1 & 1 & 0 \\
$\frac{1}{2}$ & $\frac{1}{2}$ & 1 & 0 \\
0 & 1 & 1 & 1 \\
\hline
\end{tabular}

\begin{tabular}{|c|ccc|}
\hline$\rightarrow_{9}$ & 1 & $\frac{1}{2}$ & 0 \\
\hline 1 & 1 & 1 & 0 \\
$\frac{1}{2}$ & $\frac{1}{2}$ & 1 & 0 \\
0 & 1 & $\frac{1}{2}$ & 1 \\
\hline
\end{tabular}

\footnotetext{
${ }^{7} \mathrm{~B}$ предложенном ниже перечне это импликации $\rightarrow_{1}$ и $\rightarrow_{4}$.
} 


\begin{tabular}{|c|c|c|c|c|c|c|c|c|c|c|c|}
\hline$\rightarrow_{10}$ & 1 & $\frac{1}{2}$ & 0 & $\rightarrow_{11}$ & 1 & $\frac{1}{2}$ & 0 & $\rightarrow_{12}$ & 1 & $\frac{1}{2}$ & 0 \\
\hline 1 & 1 & 1 & 0 & 1 & 1 & $\frac{1}{2}$ & 0 & 1 & 1 & $\frac{1}{2}$ & 0 \\
\hline$\frac{1}{2}$ & 1 & 1 & 0 & $\frac{1}{2}$ & $\frac{1}{2}$ & 1 & 0 & $\frac{1}{2}$ & $\frac{1}{2}$ & 1 & 0 \\
\hline 0 & 1 & $\frac{1}{2}$ & 1 & 0 & 1 & 1 & 1 & 0 & 1 & $\frac{1}{2}$ & 1 \\
\hline$\rightarrow_{13}$ & 1 & $\frac{1}{2}$ & 0 & $\rightarrow_{14}$ & 1 & $\frac{1}{2}$ & 0 & $\rightarrow_{15}$ & 1 & $\frac{1}{2}$ & 0 \\
\hline 1 & 1 & $\frac{1}{2}$ & 0 & 1 & 1 & 0 & 0 & 1 & 1 & 0 & 0 \\
\hline$\frac{1}{2}$ & 1 & 1 & 0 & $\frac{1}{2}$ & $\frac{1}{2}$ & 1 & 0 & $\frac{1}{2}$ & 1 & 1 & 0 \\
\hline 0 & 1 & $\frac{1}{2}$ & 1 & 0 & 1 & 1 & 1 & 0 & 1 & $\frac{1}{2}$ & 1 \\
\hline$\rightarrow_{16}$ & 1 & $\frac{1}{2}$ & 0 & $\rightarrow_{17}$ & 1 & $\frac{1}{2}$ & 0 & $\rightarrow_{18}$ & 1 & $\frac{1}{2}$ & 0 \\
\hline 1 & 1 & 0 & 0 & 1 & 1 & 1 & 0 & 1 & 1 & 1 & 0 \\
\hline$\frac{1}{2}$ & $\frac{1}{2}$ & 1 & 0 & $\frac{1}{2}$ & 1 & $\frac{1}{2}$ & 0 & $\frac{1}{2}$ & $\frac{1}{2}$ & $\frac{1}{2}$ & 0 \\
\hline 0 & 1 & $\frac{1}{2}$ & 1 & 0 & 1 & 1 & 1 & 0 & 1 & 1 & 1 \\
\hline$\rightarrow_{19}$ & 1 & $\frac{1}{2}$ & 0 & $\rightarrow_{20}$ & 1 & $\frac{1}{2}$ & 0 & $\rightarrow_{21}$ & 1 & $\frac{1}{2}$ & 0 \\
\hline 1 & 1 & 1 & 0 & 1 & 1 & 1 & 0 & 1 & 1 & $\frac{1}{2}$ & 0 \\
\hline$\frac{1}{2}$ & 1 & $\frac{1}{2}$ & 0 & $\frac{1}{2}$ & $\frac{1}{2}$ & $\frac{1}{2}$ & 0 & $\frac{1}{2}$ & 1 & $\frac{1}{2}$ & 0 \\
\hline 0 & 1 & $\frac{1}{2}$ & 1 & 0 & 1 & $\frac{1}{2}$ & 1 & 0 & 1 & 1 & 1 \\
\hline$\rightarrow_{22}$ & 1 & $\frac{1}{2}$ & 0 & $\rightarrow_{23}$ & 1 & $\frac{1}{2}$ & 0 & $\rightarrow_{24}$ & 1 & $\frac{1}{2}$ & 0 \\
\hline 1 & 1 & $\frac{1}{2}$ & 0 & 1 & 1 & $\frac{1}{2}$ & 0 & 1 & 1 & $\frac{1}{2}$ & 0 \\
\hline$\frac{1}{2}$ & $\frac{1}{2}$ & $\frac{1}{2}$ & 0 & $\frac{1}{2}$ & 1 & $\frac{1}{2}$ & 0 & $\frac{1}{2}$ & $\frac{1}{2}$ & $\frac{1}{2}$ & 0 \\
\hline 0 & 1 & 1 & 1 & 0 & 1 & $\frac{1}{2}$ & 1 & 0 & 1 & $\frac{1}{2}$ & 1 \\
\hline$\rightarrow_{25}$ & 1 & $\frac{1}{2}$ & 0 & $\rightarrow_{26}$ & 1 & $\frac{1}{2}$ & 0 & $\rightarrow_{27}$ & 1 & $\frac{1}{2}$ & 0 \\
\hline 1 & 1 & 0 & 0 & 1 & 1 & 0 & 0 & 1 & & 0 & 0 \\
\hline$\frac{1}{2}$ & 1 & $\frac{1}{2}$ & 0 & $\frac{1}{2}$ & $\frac{1}{2}$ & $\frac{1}{2}$ & 0 & $\frac{1}{2}$ & 1 & $\frac{1}{2}$ & 0 \\
\hline 0 & 1 & 1 & 1 & 0 & 1 & 1 & 1 & 0 & 1 & $\frac{1}{2}$ & 1 \\
\hline$\rightarrow_{28}$ & 1 & $\overline{2}$ & 0 & & & & & & & & \\
\hline 1 & 1 & 0 & 0 & & & & & & & & \\
\hline$\frac{1}{2}$ & $\frac{1}{2}$ & $\frac{1}{2}$ & 0 & & & & & & & & \\
\hline 0 & 1 & $\frac{1}{2}$ & 1 & & & & & & & & \\
\hline
\end{tabular}

Среди приведенных таблиц присутствуют таблицы, соответствующие импликациям известных трехзначных логик: $\rightarrow_{3}-$ 
импликация Лукасевича [18] в $\mathbf{Ł}_{\mathbf{3}} ; \rightarrow_{5}-$ импликация логики Бочвара [1] в $\mathbf{B}_{\mathbf{3}} ; \rightarrow_{21}$ - импликация Яськовского [17], затем появившаяся в [12] и в [5] (логика PCont); импликация $\rightarrow_{2}$ появилась независимым образом в [24] и [20] с целью сохранения сиандартой теоремы дедукции в $\mathbf{L}_{\mathbf{3}} ; \rightarrow_{25}-$ импликация Собочиньского [25], которая появляется затем в логике RM3 [10]. Две импликации, уже упоминаемые нами, следующие: $\rightarrow_{1}-$ импликация логики Гейтинга $\mathbf{G}_{\mathbf{3}}(1930$ г.) и стандартная импликация Решера $\rightarrow_{4}$ [22]. Импликация $\rightarrow_{7}$ встречается в паранепротиворечивой логике Сетте $\mathbf{P}_{\mathbf{1}}$ [23]. Подробно обо всех этих логиках см. в [2].

ОПРЕДЕЛЕНИЕ 4. Расширением логики $S$ назовем некоторую логику $S^{\prime}$, которая представляет собой пополнение исходного множества связок логики $S$ связкой, которая не может быть определена посредством исходных связок системы $S$.

Далее нас будут интересовать импликативные расширения регулярных логик Клини за счет добавления связки естественной импликации.

Поскольку в каждой регулярной логике в качестве исходной связки присутствует регулярное отрицание $\sim$, поэтому прежде чем приступить к непосредственному рассмотрению импликативных расширений регулярных логик, докажем ряд утверждений относительно систем с исходными связками $\sim$ и $\rightarrow_{i}$ $(1 \leq i \leq 28)$.

УТВЕРЖДЕНИЕ 5. Логики со связками $\sim, \rightarrow_{1} u \sim, \rightarrow_{6}$ функиионально эквивалентны.

Доказательство. Используем определения 1 и 2; доказательство утверждения следует из следующих соотношений:

$$
\begin{aligned}
& \text { (1) } p \rightarrow_{1} q=\sim q \rightarrow_{6} \sim p, \\
& \text { (2) } p \rightarrow_{6} q=\sim q \rightarrow_{1} \sim p .
\end{aligned}
$$

Q.E.D.

УТВЕРЖДЕНИЕ 6. Логики со связками $\sim, \rightarrow_{3} u \sim, \rightarrow_{12}$ функиионально эквивалентны. 


\section{Доказательство.}

(1) Заметим, логика со связками $\sim, \rightarrow_{3}$ есть трехзначная логика Лукасевича $\mathbf{E}_{\mathbf{3}}$. В силу функциональной предполноты $\mathbf{Ł}_{\mathbf{3}}[7]^{8}$, очевидно, что посредством $\sim$ и $\rightarrow_{3}$ определима импликация $\rightarrow_{12}$.

(2) Покажем, что посредством множества связок $\left\{\sim, \rightarrow_{12}\right\}$ определимы связки из множества $\left\{\sim, \rightarrow_{3}\right\}$ :

$$
p \rightarrow_{3} q=\sim\left(p \rightarrow_{12} q\right) \rightarrow_{12} \sim\left(q \rightarrow_{12} \sim p\right)
$$

Q.E.D.

УТВЕРЖКДЕНИЕ 7. Логики со связками $\sim, \rightarrow_{5} u \sim, \rightarrow_{7}$ функционально эквивалентны.

Доказательство. Доказательство утверждения следует из следующих соотношений:

$$
\begin{aligned}
& \text { (1) } p \rightarrow_{5} q=\sim q \rightarrow_{7} \sim p, \\
& \text { (2) } p \rightarrow_{7} q=\sim q \rightarrow_{5} \sim p .
\end{aligned}
$$

Q.E.D.

УТВЕРЖДЕНИЕ 8. Логики со связками $\sim, \rightarrow_{14} u \sim, \rightarrow_{15}$ функиионально эквивалентны.

Доказательство. Доказательство утверждения следует из соотношений:

$$
\begin{aligned}
& \text { (1) } p \rightarrow_{14} q=\sim q \rightarrow_{15} \sim p \\
& \text { (2) } p \rightarrow_{15} q=\sim q \rightarrow_{14} \sim p .
\end{aligned}
$$

Q.E.D.

УТВЕРЖКДЕНИЕ 9. Логики со связками $\sim, \rightarrow_{17} u \sim, \rightarrow_{25}$ функционально эквивалентны.

\footnotetext{
${ }^{8}$ Логика $L-$ функционально предполна, если она не является функционально полной, но добавление к $L$ связки, которая не выразима посредством исходных связок логики $L$, превращает $L$ с этой связкой в функционально полную логику.
} 
Доказательство. Доказательство утверждения следует из соотношений:

$$
\begin{aligned}
& \text { (1) } p \rightarrow_{17} q=\left(q \rightarrow_{25} \sim p\right) \rightarrow_{25}\left(p \rightarrow_{25} q\right), \\
& \text { (2) } p \rightarrow_{25} q=\left(\left(\sim q \rightarrow_{17} \sim p\right) \rightarrow_{17} \sim\left(p \rightarrow_{17} q\right)\right) .
\end{aligned}
$$

Q.E.D.

УТВЕРЖКДЕНИЕ 10. Логики со связками $\sim, \rightarrow_{18} u \sim, \rightarrow_{21}$ функционально эквивалентны.

Доказательство. Доказательство утверждения следует из соотношений:

$$
\begin{aligned}
& \text { (1) } p \rightarrow_{18} q=p \rightarrow_{21} \sim\left(q \rightarrow_{21} \sim p\right) \\
& \text { (2) } p \rightarrow_{21} q=\left(q \rightarrow_{18} p\right) \rightarrow_{18}\left(p \rightarrow_{18} q\right) .
\end{aligned}
$$

Q.E.D.

УТВЕРЖДДЕНИЕ 11. Логики со связками $\sim, \rightarrow_{19} u \sim, \rightarrow_{26} u$ $\sim, \rightarrow_{27}$ попарно функиионально эквивалентны.

Доказательство. Сначала покажем эквивалентность логик со связками $\sim, \rightarrow_{26}$ и $\sim, \rightarrow_{27}$. Это верно в силу следующих соотношений:

$$
\begin{aligned}
& \text { (1) } p \rightarrow_{27} q=\sim q \rightarrow_{26} \sim p, \\
& \text { (2) } p \rightarrow_{26} q=\sim q \rightarrow_{27} \sim p .
\end{aligned}
$$

Далее покажем, что логика со связками $\sim, \rightarrow_{19}$ функционально эквивалентна логике со связками $\sim, \rightarrow_{27}$. Это справедливо в силу следующих соотношений:

$$
\begin{aligned}
& \text { (1) } p \rightarrow_{27} q=p \rightarrow_{19} \sim\left(p \rightarrow_{19} \sim q\right), \\
& \text { (2) } p \rightarrow_{19} q=p \rightarrow_{27} \sim\left(p \rightarrow_{27} \sim q\right) .
\end{aligned}
$$

Q.E.D.

УТВЕРЖДЕНИЕ 12. Логики со связками $\sim, \rightarrow_{20} u \sim, \rightarrow_{22} u$ $\sim, \rightarrow 28$ попарно функиионально эквивалентны. 
Доказательство. Сначала покажем эквивалентность логик со связками $\sim, \rightarrow_{20}$ и $\sim, \rightarrow_{28}$. Это верно в силу следующих соотношений:

$$
\begin{aligned}
& \text { (1) } p \rightarrow_{20} q=\left(q \rightarrow_{28} \sim p\right) \rightarrow_{28}\left(p \rightarrow_{28} q\right), \\
& \text { (2) } p \rightarrow_{28} q=\sim\left(\left(\sim q \rightarrow_{20} \sim p\right) \rightarrow_{20} \sim\left(p \rightarrow_{20} q\right)\right) .
\end{aligned}
$$

Далее покажем, что логика со связками $\sim, \rightarrow_{20}$ функционально эквивалентна логике со связками $\sim, \rightarrow_{22}$. Это справедливо в силу следующих соотношений:

$$
\begin{aligned}
& \text { (1) } p \rightarrow_{22} q=\left(\sim p \rightarrow_{20} \sim q\right) \rightarrow_{20}\left(p \rightarrow_{20} q\right), \\
& \text { (2) } p \rightarrow_{20} q=\left(q \rightarrow_{22} \sim p\right) \rightarrow_{22}\left(p \rightarrow_{22} q\right) .
\end{aligned}
$$

Q.E.D.

УТВЕРЖДЕНИЕ 13. Посредством множества связок $\left\{\sim, \rightarrow_{i}\right\}$ $(1 \leq i \leq 16)$ выразим $J_{1}$-оператор ${ }^{9}$.

\section{Доказательство.}

$$
J_{1}(p)=\sim\left(p \rightarrow_{i} \sim p\right), \text { где } 1 \leq i \leq 16 .
$$

Q.E.D.

\section{3 Импликативные расширения регулярных логик}

\section{1 Расширения $\mathrm{K}_{3}$}

Последовательно опишем все импликативные расширения $\mathbf{K}_{\mathbf{3}}$. Напомним, $\mathbf{K}_{\mathbf{3}}$ есть логика с исходным множеством связок $\{\sim, \vee, \wedge\}$.

УТВЕРЖДЕНИЕ 14. Расширение $\mathbf{K}_{\mathbf{3}}$ посредством добавления импликации $\rightarrow_{i}(1 \leq i \leq 16)$ есть трехзначная логика Лукасевича $\mathbf{E}_{\mathbf{3}}$.

${ }^{9}$ Трехзначные $j_{i}$-операторы впервые введены в [1]:

$$
j_{i}(x)=\left\{\begin{array}{l}
1, \text { если } x=i \\
0, \text { если } x \neq i .
\end{array}\right.
$$


Доказательство. Напомним, в нашем обозначении логика Лукасевича $\mathbf{Ł}_{3}$ есть логика с исходными связками $\sim$ и $\rightarrow_{3}$.

Покажем, что логика со связками $\sim$ и $\rightarrow_{3}$ функционально эквивалентна логике со связками $\sim, \vee, \wedge, \rightarrow_{i}(1 \leq i \leq 16)$.

Это справедливо, с одной стороны, в силу функциональной предполноты логики $\mathbf{E}_{\mathbf{3}}$, с другой стороны, в силу того, что в каждой из 16 систем со связками $\left\{\sim, \vee, \wedge, \rightarrow_{i}\right\}(1 \leq i \leq 16)$ выразима импликация $\rightarrow_{3}$. Последнее обоснуем так. Расшире-

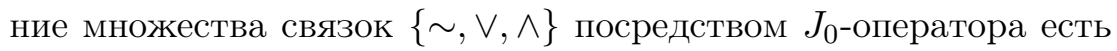
трехзначная логика Лукасевича. Это очевидно, если принять во внимание (а) в $\mathbf{L}_{\mathbf{3}}$ определимы все $J_{i}$-операторы; (б) определение импликации Лукасевича (в нашем обозначении $\rightarrow_{3}$ ) в работе [24]:

$$
p \rightarrow_{3} q=(\sim p \vee q) \vee \sim J_{0}(\sim p \wedge q) .
$$

Таким образом, достаточно посредством множества связок $\left\{\sim, \vee, \wedge, \rightarrow_{i}\right\}(1 \leq i \leq 16)$ определить $J_{0}$-оператор. А это имеет место в силу ранее доказанного утверждения 13, а также соотношений:

$$
J_{1}(p)=J_{0}(\sim p) \text { и } J_{0}(p)=J_{1}(\sim p) .
$$

Итак, утверждение 14 доказано.

Q.E.D.

УТВЕРЖДЕНИЕ 15. Расширение $\mathbf{K}_{\mathbf{3}}$ посредством $\rightarrow_{i}(i \in$ $\{17,18\})$ есть логика PCont.

Доказательство. Покажем, что логика со связками $\sim, \vee, \wedge, \rightarrow_{i}$ $(i \in\{17,18\})$ функционально эквивалентна логике со связками $\sim, \vee, \wedge, \rightarrow_{21}$.

В работе [11] доказано, что логика PCont функционально эквивалентна логике $\mathbf{R M 3}$, т.е. в нашем обозначении логика со множествами связок $\left\{\sim, \vee, \wedge, \rightarrow_{21}\right\}$ и $\left\{\sim, \vee, \wedge, \rightarrow_{25}\right\}$ функционально эквивалентны.

Доказательство утверждения 15 следует из вышесказанного, а также из ранее обоснованных утверждений 9 и $10 . \quad$ Q.E.D.

УТВЕРЖДЕНИЕ 16. Расширение $\mathbf{K}_{\mathbf{3}}$ посредством $\rightarrow_{i}(i \in$ $\{19,20,22,26,27,28\})$ есть логика PCont. 
Доказательство. В силу ранее доказанных утверждений 11 и 12, для доказательства утверждения 16 достаточно показать, что:

(1) логика со множеством исходных связок $\left\{\sim, \vee, \wedge, \rightarrow_{19}\right\}$ функционально эквивалентна логике со множеством связок $\{\sim, \vee, \wedge$, $\left.\rightarrow_{20}\right\}$;

(2) логика со связками $\sim, \vee, \wedge, \rightarrow_{19}$ функционально эквивалентна логике со связками $\sim, \vee, \wedge, \rightarrow 18$. (Последняя в силу утверждения 15 есть логика PCont.)

Итак, (1) имеет место, т.к.

$$
\begin{aligned}
& p \rightarrow_{19} q=\left(p \rightarrow_{20} q\right) \vee q, \\
& p \rightarrow_{20} q=(p \vee \sim p) \vee\left(p \rightarrow_{20} q\right) .
\end{aligned}
$$

(2) справедливо, т.к.:

$$
\begin{aligned}
& p \rightarrow_{19} q=\left(q \rightarrow_{18} p\right) \rightarrow_{18}\left(p \rightarrow_{18} q\right) \text { и } \\
& p \rightarrow_{18} q=\left(\left(p \rightarrow_{19} q\right) \wedge p\right) \rightarrow_{19}(p \wedge \sim q) .
\end{aligned}
$$

Таким образом, утверждение 16 доказано.

Q.E.D.

УТВЕРЖДЕНИЕ 17. Расширение $\mathbf{K}_{\mathbf{3}}$ посредством $\rightarrow_{i}(i \in$ $\{23,24\})$ есть логика PCont.

Доказательство. Для доказательства утверждения 17 достаточно показать, что:

(1) логика со связками $\sim, \vee, \wedge, \rightarrow_{23}$ функционально эквивалентна логике со связками $\sim, \vee, \wedge, \rightarrow_{24}$;

(2) логика со связками $\sim, \vee, \wedge, \rightarrow_{23}$ функционально эквивалентна логике со связками $\sim, \vee, \wedge, \rightarrow_{21}$ (PCont).

(1) справедливо в силу следующих соотношений:

$$
\begin{aligned}
& p \rightarrow_{23} q=\left(p \rightarrow_{24} q\right) \vee q \text { и } \\
& p \rightarrow_{24} q=\left(p \rightarrow_{23} q\right) \wedge(p \vee \sim p) .
\end{aligned}
$$

(2) справедливо в силу соотношений:

$$
\begin{aligned}
& p \rightarrow_{23} q=\left(p \rightarrow_{21} q\right) \wedge(q \vee \sim q) \text { и } \\
& p \rightarrow_{21} q=\left(p \rightarrow_{23} q\right) \vee \sim\left(q \rightarrow_{23}(p \wedge(\sim q \wedge q))\right) .
\end{aligned}
$$

Итак, утверждение 17 доказано.

Q.E.D. 
Таким образом, при рассмотрении расширений сильной регулярной логики Клини $\mathbf{K}_{\mathbf{3}}$ посредством класса естественных импликаций получили, с одной стороны, класс логик, по функииональным свойствам эквивалентных логике Лукасевича $\mathbf{E}_{\mathbf{3}}$, с другой стороны, класс логик, функиионально эквивалентных rozuкe PCont.

Таблица разбиений всех 28 импликаций на классы выглядит следующим образом:

\begin{tabular}{|c|c|}
\hline $\mathbf{E}_{\mathbf{3}}$ & PCont \\
\hline$\rightarrow_{i}$ & $\rightarrow_{i}$ \\
$(1 \leq i \leq 16)$ & $(17 \leq i \leq 28)$ \\
\hline
\end{tabular}

Заметим, что расширение PCont константой 1 приводит к другой известной паранепротиворечивой логике $\mathbf{J}_{\mathbf{3}}$, которая по функциональным свойствам есть $\mathbf{E}_{\mathbf{3}}$. Тогда расширения $\mathbf{K}_{\mathbf{3}}$ можно представить так:

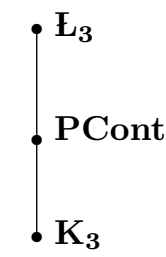

Рис. 1

\section{2 Расширения $\mathrm{K}_{3}$}

Последовательно рассмотрим все импликативные расширения

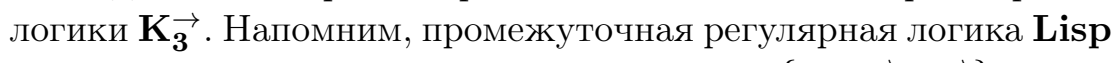
есть логика с исходным множеством связок $\{\sim, \vee \rightarrow, \wedge \rightarrow\}$.

УТВЕРЖДЕНИЕ 18 . Расширение $\mathbf{K}_{\mathbf{3}} \rightarrow$ посредством добавления импликации $\rightarrow_{i}(1 \leq i \leq 16)$ есть трехзначная логика Лукасевича $\mathbf{E}_{\mathbf{3}}$.

Доказательство. Напомним, в нашем обозначении логика Лукасевича $\mathbf{Ł}_{3}$ есть логика с исходными связками $\sim$ и $\rightarrow_{3}$.

Покажем, что логика со связками $\sim$ и $\rightarrow_{3}$ функционально эквивалентна логике со связками $\sim, \vee \rightarrow, \wedge \rightarrow, \rightarrow_{i}(1 \leq i \leq 16)$. 
Это справедливо, с одной стороны, в силу функциональной предполноты логики $\mathbf{E}_{\mathbf{3}}$, с другой стороны, в силу того, что в каждой из 16 систем со связками $\left\{\sim, \vee \rightarrow, \wedge \rightarrow, \rightarrow{ }_{i}\right\}(1 \leq i \leq 16)$ выразима импликация $\rightarrow_{3}$. Последнее очевидно имеет место, если принять во внимание следующее:

(a) расширение $\mathbf{K}_{\mathbf{3}} \rightarrow$ посредством любого из трех $J_{i}$-операторов представляет собой трехзначную логику Лукасевича [6];

(б) ранее доказанное утверждение 13.

Таким образом, утверждение 18 доказано.

Q.E.D.

УТВЕРЖДЕНИЕ 19. Расширение $\mathbf{K}_{\mathbf{3}}$ посредством $\rightarrow_{i}(i \in$ $\{17,18,21,25\})$ есть логика PCont.

Доказательство. В силу ранее доказанных утверждений 9 и 10, а также утверждений о том, что расширение $\mathbf{K}_{\mathbf{3}}$ посредством $\rightarrow_{i}(i \in\{17,18,21,25\})$ есть логика PCont, и учитывая, что $\mathbf{K}_{\mathbf{3}} \subset \mathbf{K}_{\mathbf{3}}$, для доказательства утверждения 19 достаточно посредством каждого множества связок $\left\{\sim, \vee \rightarrow, \wedge \rightarrow, \rightarrow_{21}\right\}$ и $\left\{\sim, \vee^{\rightarrow}, \wedge^{\rightarrow}, \rightarrow_{25}\right\}$ определить сильную регулярную дизъюнкцию $\checkmark$. Это можно сделать, например, так:

$$
\begin{aligned}
& p \vee q=\left(\sim q \rightarrow_{21} p\right) \vee \rightarrow\left(\sim p \rightarrow_{21} q\right) \text { и } \\
& p \vee q=\left(\left(\sim p \rightarrow_{25} q\right) \vee \rightarrow p\right) \vee \rightarrow q .
\end{aligned}
$$

Утверждение 19 доказано.

Q.E.D.

УТВЕРЖДЕНИЕ 20. Расширение $\mathbf{K}_{\mathbf{3}}$ посредством $\rightarrow_{i}(i \in$ $\{19,20,22,26,27,28\})$ есть логика PCont.

Доказательство. В силу ранее доказанных утверждений 11 и 12 , а также утверждений о том, что расширение $\mathbf{K}_{\mathbf{3}}$ посредством $\rightarrow_{i}(i \in\{19,20,22,26,27,28\})$ есть логика PCont, и учитывая, что $\mathbf{K}_{\mathbf{3}} \subset \mathbf{K}_{\mathbf{3}}$, для доказательства утверждения 20 достаточно посредством каждого множества связок, например, $\left\{\sim, \vee^{\rightarrow}, \wedge^{\rightarrow}, \rightarrow_{20}\right\}$ и $\left\{\sim, \vee^{\rightarrow}, \wedge^{\rightarrow}, \rightarrow_{27}\right\}$, определить сильную регулярную дизъюнкцию $\vee$. Это можно сделать, например, так:

$$
\begin{aligned}
& p \vee q=\left(\left(p \rightarrow_{20} \sim q\right) \vee \rightarrow q\right) \rightarrow_{20}(p \vee \rightarrow q) \text { и } \\
& p \vee q=\left(\left(\sim p \rightarrow_{27} q\right) \vee^{\rightarrow} p\right) \vee^{\rightarrow} q .
\end{aligned}
$$

Утверждение 20 доказано.

Q.E.D. 
УТВЕРЖДЕНИЕ 21. Расширение $\mathbf{K}_{\mathbf{3}} \rightarrow$ посредством импликации $\rightarrow_{23}$ функиионально эквивалентно расширению $\mathbf{K}_{\mathbf{3}} \rightarrow$ посредством импликачии $\rightarrow_{24}$.

Доказательство. Доказательство утверждения следует из следующих соотношений:

$$
\begin{aligned}
& \text { (1) } p \rightarrow_{23} q=q \vee \rightarrow\left(p \rightarrow_{24} q\right) \text { и } \\
& \text { (2) } p \rightarrow_{24} q=\left(\left(p \rightarrow_{23} q\right) \wedge^{\rightarrow} p\right) \vee \rightarrow\left(p \rightarrow_{23} q\right) .
\end{aligned}
$$

Утверждение 21 доказано.

Q.E.D.

Далее, определим, что представляет с функциональной точки зрения логика со связками $\sim, \vee \rightarrow, \wedge \rightarrow, \rightarrow_{23}$. Ранее было показано (утверждение 17), что логика со связками $\sim, \vee, \wedge, \rightarrow_{23}$ есть логика PCont. Тогда, учитывая, что $\mathbf{K}_{\mathbf{3}} \subset \mathbf{K}_{\mathbf{3}}$, можем утверждать, что логика со связками $\sim, \vee^{\rightarrow}, \wedge \rightarrow, \rightarrow_{23}$ функционально вложима в логику PCont. Однако не можем говорить о функциональной эквивалентности этих логик, поскольку если бы это имело место, то посредством множества связок $\left\{\sim, \vee \rightarrow, \wedge \rightarrow, \rightarrow_{23}\right\}$ была бы выразима, например, импликация $\rightarrow_{25}$ или, что аналогично, дизъюнкция $\vee_{25}$ (где $p \rightarrow_{25} q=$ $\sim p \vee_{25} q$ ). Обратимся к таблице для $\vee_{25}$, очевидно, дизъюнкция $\vee_{25}$ коммутативна: $1 \vee_{25} \frac{1}{2}=1$ и $\frac{1}{2} \vee_{25} 1=1$. Подобную дизъюнкцию посредством связок $\sim, \vee \rightarrow, \wedge \rightarrow, \rightarrow_{23}$ определить невозможно в силу их некоммутативности ${ }^{10}$. Подобное рассуждение дает основание для следующего утверждения:

УТВЕРЖДЕНИЕ 22. Расширение $\mathbf{K}_{\mathbf{3}}$ посредством $\rightarrow_{i}$ $(i \in\{23,24\})$ не является функиионально эквивалентным лоәике PCont.

Строгое доказательство утверждения 22 остается открытой проблемой.

Обозначим логику со связками $\sim, \vee \rightarrow, \wedge \rightarrow, \rightarrow_{23}$ (а также функционально эквивалентные ей логики) как $\mathbf{T}^{2}$.

\footnotetext{
${ }^{10}$ Очевидно, логика со связками $\sim, \vee \rightarrow, \wedge \rightarrow, \rightarrow 23$ функционально эквивалентна логике со связками $\sim, \vee^{\rightarrow}, \wedge \rightarrow, \vee_{23}$ (где $p \vee_{23} q=\sim p \rightarrow_{23} q$ ). Дизъюнкция $\vee_{23}$, так же как и связки $\vee^{\rightarrow}, \wedge^{\rightarrow}$, является некоммутативной.
} 
Таким образом, при рассмотрении расширений промежуточной регулярной логики Клини $\mathbf{K}_{\mathbf{3}}$ посредством класса естественных импликаций получили, три класса логик: с одной стороны, класс логик, по функииональным свойствам эквивалентных логике Лукасевича $\mathbf{E}_{\mathbf{3}}$, с другой стороны, класс систем, эквивалентных логике PCont, а также класс логик, эквивалентных логике $\mathbf{T}^{2}$. Эта логика встречается впервые.

Таблица разбиений всех 28 импликаций на классы выглядит следующим образом:

\begin{tabular}{|c|c|c|}
\hline $\mathbf{L}_{\mathbf{3}}$ & PCont & $\mathbf{T}^{\mathbf{2}}$ \\
\hline$\rightarrow_{i}$ & $\rightarrow_{i}$ & $\vec{\rightarrow}_{i}$ \\
$(1 \leq i \leq 16)$ & $(i \in\{17,18,19,20,21$, \\
& $22,25,26,27,28\})$ & $(i \in\{23,24\})$ \\
\hline
\end{tabular}

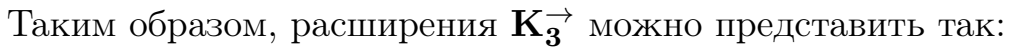

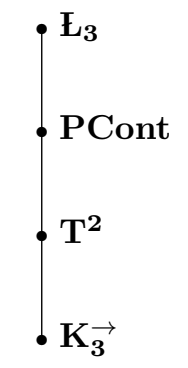

Рис. 2

\section{3 Расширения $\mathrm{K}_{3}^{\mathrm{w}}$}

Рассмотрим все импликативные расширения логики $\mathbf{K}_{\mathbf{3}}^{\mathbf{w}}$. Напомним, $\mathbf{K}_{\mathbf{3}}^{\mathbf{w}}$ есть логика с исходным множеством связок $\{\sim, \cup, \cap\}$.

Учитывая ранее доказанное утверждение 6, а также тот факт, что логика Лукасевича $\mathbf{Ł}_{\mathbf{3}}$ есть логика с исходными связками $\sim$ и $\rightarrow_{3}$, очевидно, что расширение $\mathbf{K}_{\mathbf{3}}^{\mathbf{w}}$ посредством добавления импликации $\rightarrow_{i}(i \in\{3,12\})$ есть трехзначная логика Лукасевича $\mathbf{E}_{3}$.

Для дальнейшего исследования нам потребуется следующее утверждение. 
УТВЕРЖДЕНИЕ 23. Расширение $\mathbf{K}_{\mathbf{3}}^{\mathbf{w}}$ посредством добавления $J_{1}$-оператора или $J_{0}$-оператор есть логика $\mathbf{B}_{\mathbf{3}}$.

Доказательство. Напомним, в связи с проблемой анализа логических антиномий Д.А. Бочвар [1] построил трехзначную логику $\mathbf{B}_{\mathbf{3}}$, которая имеет следующие исходные связки $\sim, \cap, \vdash$, где $\sim$ и $\cap$ определяются так же, как в слабой логике Клини $\mathbf{K}_{\mathbf{3}}^{\mathbf{w}}$, а связка $\vdash$ называется внешним утверждением и есть не что иное, как $J_{1}$-оператор. Таким образом, учитывая, что $J_{1}(p)=J_{0}(\sim p)$ и $J_{0}(p)=J_{1}(\sim p)$, утверждение 23 доказано. Q.E.D.

УТВЕРЖДДНИЕ 24. Расширение $\mathbf{K}_{\mathbf{3}}^{\mathbf{w}}$ посредством $\rightarrow_{i}(i \in$ $\{4,5,7\})$ есть логика $\mathbf{B}_{\mathbf{3}}$.

Доказательство. В работе [8, с. 401] показано, что логика со связками $\sim, \cup, \cap, \rightarrow_{5}$ есть $\mathbf{B}_{\mathbf{3}}$.

Тогда, учитывая ранее доказанные утверждение 7, 13, 23 для доказательства утверждения 24 достаточно показать, что логика со связками $\sim, \cup, \cap, \rightarrow_{4}$ функционально вложима в логику со связками $\sim, \cup, \cap, \rightarrow_{5}$. Это следует из соотношения:

$$
p \rightarrow_{4} q=\left(p \rightarrow_{5} q\right) \cap\left(\sim q \rightarrow_{5} \sim p\right) .
$$

Утверждение 24 доказано.

Q.E.D.

УТВЕРЖДДНИЕ 25. Расширение $\mathbf{K}_{\mathbf{3}}^{\mathbf{w}}$ посредством $\rightarrow_{i}(i \in$ $\{18,19,20,21,22,26,27,28\})$ eсть логика PCont.

Доказательство. В силу ранее доказанных утверждений 10 , 11,12 , а также утверждений о том, что расширение $\mathbf{K}_{3} \rightarrow$ посредством $\rightarrow_{i}(i \in\{18,19,20,21,22,26,27,28\})$ есть логика PCont, и учитывая тот факт, что $\mathbf{K}_{\mathbf{3}}^{\mathbf{w}} \subset \mathbf{K}_{\mathbf{3}}$, для доказательства утверждения 25 достаточно посредством каждого множества связок, например, $\left\{\sim, \cup, \cap, \rightarrow_{19}\right\},\left\{\sim, \cup, \cap, \rightarrow_{20}\right\}$ и $\left\{\sim, \cup, \cap, \rightarrow_{21}\right\}$, определить регулярную дизъюнкцию $\vee \rightarrow$. Это можно сделать, например, так:

$$
\begin{aligned}
& p \vee \rightarrow q=\left(\left(p \rightarrow_{19} q\right) \cap\left(\sim q \rightarrow_{19} p\right)\right) \cup p, \\
& p \vee \rightarrow q=\left(\sim p \rightarrow_{20} q\right) \cup p, \\
& p \vee \rightarrow q=\left(\sim p \rightarrow_{21} q\right) \cup p .
\end{aligned}
$$

Утверждение 25 доказано.

Q.E.D. 
УТВЕРЖДЕНИЕ 26. Расширение $\mathbf{K}_{\mathbf{3}}^{\mathbf{w}}$ посредством добавления импликачии $\rightarrow_{i}(i \in\{1,2,6,8,9,10,11,14,15,16\})$ есть трехзначная логика Лукасевича $\mathbf{L}_{\mathbf{3}}$.

Доказательство. Покажем, что логика со связками $\sim$ и $\rightarrow_{3}\left(\mathbf{E}_{\mathbf{3}}\right)$ функционально эквивалентна логике со связками $\sim, \cup, \cap, \rightarrow_{i}$ $(i \in\{1,2,6,8,9,10,11,14,15,16\})$.

Это справедливо, с одной стороны, в силу функциональной предполноты логики $\mathbf{E}_{\mathbf{3}}$, с другой стороны, в силу того, что в каждой из 10 систем со связками $\sim, \cup, \cap, \rightarrow_{i}(i \in\{1,2,6,8,9,10$, $11,14,15,16\})$ выразима импликация $\rightarrow_{3}$. Последнее имеет место, если принять во внимание ранее доказанные утверждения 5 и 8, а также следующие выразимости ${ }^{11}$ :

$$
\begin{aligned}
& p \rightarrow_{3} q=\left(p \rightarrow_{i} q\right) \cap\left(\sim q \rightarrow_{i} \sim p\right), \text { где } i \in\{1,2\} ; \\
& p \rightarrow_{3} q=\left(\left(\sim q \rightarrow_{i} p\right) \cup\left(p \rightarrow_{i} \sim q\right)\right) \cap\left(p \rightarrow_{5} q\right), \text { где } i \in\{8,11,14\} ; \\
& p \rightarrow_{3} q=\sim\left(q \rightarrow_{i} p\right) \cup\left(p \rightarrow_{5} q\right), \text { где } i \in\{9,16\} ; \\
& p \rightarrow_{3} q=\left(\left(\sim p \rightarrow_{10} q\right) \cup\left(q \rightarrow_{10} \sim p\right)\right) \cap\left(p \rightarrow_{5} q\right) .
\end{aligned}
$$

Таким образом, утверждение 26 доказано.

Q.E.D.

УТВЕРЖДДЕНЕ 27. Расширение $\mathbf{K}_{\mathbf{3}}^{\mathbf{w}}$ посредством $\rightarrow_{24}$ функционально эквивалентно логике $\mathbf{T}^{2}$, т.е. логике со связками $\sim$, $\vee^{\rightarrow}, \wedge \rightarrow, \rightarrow_{i}(i \in\{23,24\})$.

Доказательство. Поскольку $\mathbf{K}_{\mathbf{3}}^{\mathbf{w}} \subset \mathbf{K}_{\mathbf{3}} \rightarrow$ и логика со связками $\sim, \vee^{\rightarrow}, \wedge \rightarrow, \rightarrow_{24}$ есть логика $\mathbf{T}^{\mathbf{2}}$, то для доказательства утверждения 27 достаточно посредством связок $\sim, \cup, \cap, \rightarrow_{24}$ выразить регулярную дизъюнкцию $\vee \rightarrow$. Это можно сделать, например, так:

$$
p \vee \rightarrow q=\left(\sim\left(\sim p \rightarrow_{24} q\right) \rightarrow_{24}\left(\left(q \rightarrow_{24} \sim p\right) \cap p\right)\right) \cup p .
$$

Утверждение 27 доказано.

Q.E.D.

Далее, в работе [15] рассмотрены алгебраические свойства трехзначной логики бессмысленности - логики $\mathbf{Z}$. Исходные

\footnotetext{
${ }^{11}$ При выражении $\rightarrow 3$ в некоторых случаях будет использована связка $\rightarrow_{5}$. Очевидно, в силу ранее обоснованных утверждений 13 и 23, что $\rightarrow_{5}$ определима посредством множества связок $\sim, \cup, \cap, \rightarrow_{i}(i \in$ $\{1,2,6,8,9,10,11,14,15,16\})$.
} 
связки логики $\mathbf{Z}$ : инволюция $\sim$, слабая регулярная конъюнкция $\cap$ и дизъюнкция трехзначной логики Эббингауза $\vee^{e}[13]^{12}$. Другими словами, логика $\mathbf{Z}$ есть расширение слабой регулярной логики Клини посредством добавления дизъюнкции Эббингауза. Однако это аналогично тому, что к $\mathbf{K}_{3}^{\mathbf{w}}$ добавить импликацию $\rightarrow_{25}$, поскольку

$$
p \rightarrow{ }_{25} q=\sim p \vee^{e} q
$$

Тогда, учитывая данное рассуждение, а также ранее доказанное утверждение 9, справедливо следующее:

УТВЕРЖДЕНИЕ 28. Расширение $\mathbf{K}_{\mathbf{3}}^{\mathbf{w}}$ посредством добавления импликации $\rightarrow_{i}(i \in\{17,25\})$ есть логика $\mathbf{Z}$.

Возникает вопрос о соотношении логики $\mathbf{Z}$ с другими импликативными расширениями логики $\mathbf{K}_{\mathbf{3}}^{\mathbf{w}}$. В силу ранее доказанного утверждения 25 , а также предложенного А.А. Солощенковым определения $\rightarrow_{25}$ посредством $\sim$ и $\rightarrow_{21}$ :

$$
p \rightarrow_{25} q=\sim\left(p \rightarrow_{21} q\right) \rightarrow_{21} \sim\left(q \rightarrow_{21} p\right),
$$

можем говорить о том, что логика $\mathbf{Z}$ функционально вложима в логику PCont. C другой стороны, логика PCont не вложима в логику Z. Это следует из работы [15], поскольку в логике $\mathbf{Z}$ не определима сильная регулярная дизъюнкция (конъюнкция).

Далее, рассмотрим расширение $\mathbf{K}_{\mathbf{3}}^{\mathbf{w}}$ посредством добавления $\rightarrow 13$. Для удобства логику со связками $\sim, \cup, \cap, \rightarrow_{13}$ обозначим $\mathbf{T}^{3}$.

В силу ранее доказанных утверждений 13 и 23, очевидно, что логика Бочвара $\mathbf{B}_{3}$ функционально вложима в логику $\mathbf{T}^{3}$. Однако $\mathbf{T}^{\mathbf{3}}$ не вложима в $\mathbf{B}_{\mathbf{3}}{ }^{13}$.

Можно доказать следующее утверждение.

УТВЕРЖДЕНИЕ 29. Логика $\mathbf{Z}$ функиионально вложима в лоәику $\mathbf{T}^{\mathbf{3}}$.

Доказательство. Доказательство следует из соотношения:

\footnotetext{
${ }^{12}$ Напомним, что логика Эббингауза $\mathbf{E}_{3}$ представляет собой расширение логики Бочвара $\mathbf{B}_{3}$ за счет добавления дизъюнкции $\vee^{e}$.

${ }^{13}$ Из построения нормальных форм ( $I$ - $J$-с.д.н.ф.) для логики $\mathbf{B}_{3}[8]$ следует, что импликация $\rightarrow 13$ не определима в $\mathbf{B}_{\mathbf{3}}$.
} 


$$
p \rightarrow_{25} q=\left(p \rightarrow_{13} q\right) \rightarrow_{13}\left(\left(\sim q \rightarrow_{13} \sim p\right) \rightarrow_{13} p\right) .
$$

Утверждение 29 доказано.

Q.E.D.

В то же время, $\mathbf{T}^{\mathbf{3}}$ не вложима в $\mathbf{Z}$, поскольку все связки логики $\mathbf{Z}$ сохраняют значение $\frac{1}{2}$ при значении аргументов $\frac{1}{2}$, в то время как свойства импликации логики $\mathbf{T}^{\mathbf{3}}$ таковы, что $\frac{1}{2} \rightarrow_{13} \frac{1}{2}=1$.

C другой стороны, $\mathbf{T}^{\mathbf{3}}$ функционально вложима в $\mathbf{E}_{\mathbf{3}}$. Однако $\mathbf{E}_{\mathbf{3}}$ не вложима в $\mathbf{T}^{\mathbf{3}}$, в противном случае, в $\mathbf{T}^{\mathbf{3}}$ была бы выразима дизъюнкция $\vee$, которая есть $\max (x, y): 1 \vee \frac{1}{2}=1$ и $\frac{1}{2} \vee 1=1$. Дизъюнкцию $\vee$ посредством связок $\sim, \cup, \cap, \rightarrow 13$ определить невозможно в силу некоммутативности $\vee_{13}: 1 \vee_{13} \frac{1}{2}=\frac{1}{2}$, $\frac{1}{2} \vee_{13} 1=1$ и $\frac{1}{2} \vee_{13} 0=0,0 \vee_{13} \frac{1}{2}=\frac{1}{2} \cdot{ }^{14}$

Далее, рассмотрим расширение $\mathbf{K}_{\mathbf{3}}^{\mathbf{w}}$ посредством добавления $\rightarrow_{23}$. Для удобства логику со связками $\sim, \cup, \cap, \rightarrow_{23}$ обозначим $\mathbf{T}^{\mathbf{1}}$. Относительно логики $\mathbf{T}^{\mathbf{1}}$ докажем утверждения 30-32.

УТВЕРЖДЕНИЕ 30. Логика $\mathbf{T}^{\mathbf{1}}$ функционально вложима в логику $\mathbf{Z}$.

Доказательство. Для доказательства достаточно посредством связок $\sim, \cup, \cap, \rightarrow_{25}$ определить импликацию $\rightarrow_{23}$. Это можно сделать, например, так:

$$
p \rightarrow_{23} q=\left(p \rightarrow_{25} q\right) \cup q .
$$

Утверждение 30 доказано.

Q.E.D.

УТВЕРЖДЕНИЕ 31. Логика $\mathbf{T}^{\mathbf{1}}$ функционально вложима в логику $\mathbf{T}^{2}$.

Доказательство. Для доказательства достаточно посредством связок $\sim, \cup, \cap, \rightarrow_{24}$ определить импликацию $\rightarrow_{23}$. Это можно сделать, например, так:

$$
\left.p \rightarrow_{23} q=\left(\left(p \rightarrow_{24} q\right) \rightarrow_{24}(\sim q \cap q)\right) \cup q\right) .
$$

Утверждение 31 доказано.

Q.E.D.

\footnotetext{
${ }^{14}$ Очевидно, логика со связками $\sim, \cup, \cap, \rightarrow 13$ функционально эквивалентна логике со связками $\sim, \cup, \cap, \vee_{13}$ (где $p \vee_{13} q=\sim p \rightarrow_{13} q$ ).
} 
УТВЕРЖДЕНИЕ 32. Логика $\mathbf{T}^{\mathbf{1}}$ функционально вложима в логику $\mathbf{B}_{\mathbf{3}}$.

Доказательство. Для доказательства достаточно посредством связок $\sim, \cup, \cap, \rightarrow_{5}$ определить импликацию $\rightarrow_{23}$. Это можно сделать, например, так:

$$
p \rightarrow_{23} q=\left(\sim q \rightarrow_{5} \sim p\right) \cup q .
$$

Утверждение 32 доказано.

Q.E.D.

С другой стороны, свойства связок логики $\mathbf{T}^{\mathbf{1}}$ таковы, что ни $\mathbf{Z}$, ни $\mathbf{T}^{\mathbf{2}}$, ни $\mathbf{B}_{\mathbf{3}}$ не вложимы в $\mathbf{T}^{\mathbf{1}}$.

$\mathbf{B}_{\mathbf{3}} \not \subset \mathbf{T}^{\mathbf{1}}$, поскольку знаем, что в $\mathbf{B}_{\mathbf{3}}$ выразимы все $J_{i}$-операторы, с другой стороны, очевидно, что в $\mathbf{T}^{\mathbf{1}}$ нет ни одного $J_{i^{-}}$ оператора.

$\mathbf{T}_{\mathbf{2}} \not \subset \mathbf{T}^{\mathbf{1}}$, т.к. в противном случае, учитывая доказанные утверждения 31 и 32 , получили бы, что $\mathbf{T}^{\mathbf{2}}$ и $\mathbf{T}^{\mathbf{1}}$ функционально эквивалентны и $\mathbf{T}^{\mathbf{2}} \subset \mathbf{B}_{\mathbf{3}}$. Однако $\mathbf{T}_{\mathbf{2}} \not \subset \mathbf{B}_{\mathbf{3}}$, поскольку из построения нормальных форм ( $I$ - $J$-с.д.н.ф.) для логики $\mathbf{B}_{\mathbf{3}}[8]$ следует, что импликация $\rightarrow_{24}$ (импликация логики $\mathbf{T}^{\mathbf{2}}$ ) не определима в $\mathbf{B}_{\mathbf{3}}$.

$\mathbf{Z} \not \subset \mathbf{T}^{\mathbf{1}}$, т.к. в противном случае, учитывая доказанные утверждения 30 и 32 , получили бы, что $\mathbf{Z}$ и $\mathbf{T}^{\mathbf{1}}$ функционально эквивалентны и $\mathbf{Z} \subset \mathbf{B}_{\mathbf{3}}$. Однако $\mathbf{Z} \not \subset \mathbf{B}_{\mathbf{3}}$, поскольку из построения нормальных форм ( $I$ - $J$-с.д.н.ф.) для логики $\mathbf{B}_{\mathbf{3}}[8]$ следует, что импликация $\rightarrow_{21}$ (импликация логики $\mathbf{Z}$ ) не определима в $\mathbf{B}_{\mathbf{3}}$.

Итак, при рассмотрении расширений слабой регулярной логики Клини $\mathbf{K}_{\mathbf{3}}^{\mathbf{w}}$ посредством класса естественных импликаций получили семь логик. Эти логики назовем базовыми. Таблица разбиений всех 28 импликаций на классы выглядит следующим образом:

\begin{tabular}{|c|c|c|c|c|c|c|}
\hline $\mathbf{E}_{\mathbf{3}}$ & $\mathbf{P C o n t}$ & $\mathbf{B}_{\mathbf{3}}$ & $\mathbf{Z}$ & $\mathbf{T}^{\mathbf{1}}$ & $\mathbf{T}^{\mathbf{2}}$ & $\mathbf{T}^{\mathbf{3}}$ \\
\hline$\rightarrow_{i}$ & $\rightarrow_{i}$ & $\rightarrow_{i}$ & $\rightarrow_{i}$ & $\rightarrow_{23}$ & $\rightarrow_{24}$ & $\rightarrow_{13}$ \\
$(i \in\{1,2,3,6$, & $(i \in\{18,19$, & $(i \in\{4,5,7\})$ & $(i \in\{17,25\})$ & & & \\
$8,9,10,11$, & $20,21,22$, & & & & & \\
$12,14,15,16\})$ & $26,27,28\})$ & & & & & \\
\hline
\end{tabular}

Решетка базовых трехзначных логик весьма примечательна: 


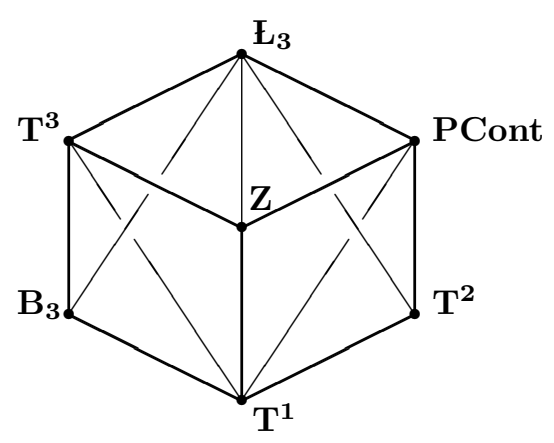

Рис. 3

Итак, семь базовых логик образуют решетку по отношению функционального вложения одной логики в другую. Это отношение является отношением порядка, поскольку оно рефлексивно, антисимметрично и транзитивно. K этому стоит добавить, что свойства связок $\cup$ и $\cap$ таковы, что они образуют дистрибутивную квази-решетку (т.е. решетку без законов поглощения), а вместе со связкой выполняют законы Де Моргана.

Важно отметить, что расширения $\mathbf{K}_{\mathbf{3}}^{\mathbf{w}}$ не образуют булеву решетку, поскольку теоретико-множественное объединение множеств связок логики $\mathbf{B}_{\mathbf{3}}$ и логики $\mathbf{T}^{\mathbf{2}}$ дает трехзначную логику $\mathbf{E}_{\mathbf{3}}$. Посредством множества связок $\left\{\sim, \cup, \cap, \rightarrow_{5}, \rightarrow_{24}\right\}^{15}$ можно определить импликацию Лукасевича $\left(\rightarrow_{3}\right)$, например, так:

$p \rightarrow_{3} q=\left(\left(q \rightarrow_{24} J_{1}(p)\right) \cap\left(p \rightarrow_{24}\left(J_{0}(q) \cap J_{\frac{1}{2}}(q)\right)\right) \cup\left(J_{1}(q) \cup J_{\frac{1}{2}}(q)\right)\right.$.

Далее, особо отметим логики $\mathbf{T}^{\mathbf{1}}, \mathbf{T}^{\mathbf{2}}, \mathbf{T}^{\mathbf{3}}$, ранее не встречавшиеся в литературе. Они оказались некоммутативными, если мы стандартным образом (посредством $\sim$ и $\left.\rightarrow_{i}(i \in\{23,24,13\})\right)$ определим в них дизъюнкции.

$\mathbf{T}^{\mathbf{1}}$ с функциональной точки зрения является самым слабым расширением логики $\mathbf{K}_{\mathbf{3}}^{\mathbf{w}}$. В работе [9] описаны 11 предполных классов логики Бочвара $\mathbf{B}_{\mathbf{3}}$, и логика $\mathbf{T}^{\mathbf{1}}$ является одним из них (класс всех внутренних функций).

\section{4 Решетка $\mathrm{L}\left(\mathrm{K}_{3}^{\mathrm{w}}\right)$ и другие трехзначные логики}

Однако возникает вопрос, почему в приведенной классификации расширений слабой регулярной логики Клини $\mathbf{K}_{\mathbf{3}}^{\mathbf{w}}$ отсутствуют

\footnotetext{
${ }^{15}$ Напомним, посредством связок $\sim, \cup, \cap, \rightarrow_{5}$ выразимы все $J_{i}$-операторы.
} 
логика Холдена $\mathbf{H}_{3}[16]^{16}$ и логика Эббингауза $\mathbf{E}_{\mathbf{3}}$ [13], в основе которых также лежит $\mathbf{K}_{\mathbf{3}}^{\mathbf{w}}$. На рис. 4 приведем решетку $\mathbf{L}\left(\mathbf{K}_{\mathbf{3}}^{\mathbf{w}}\right)$.

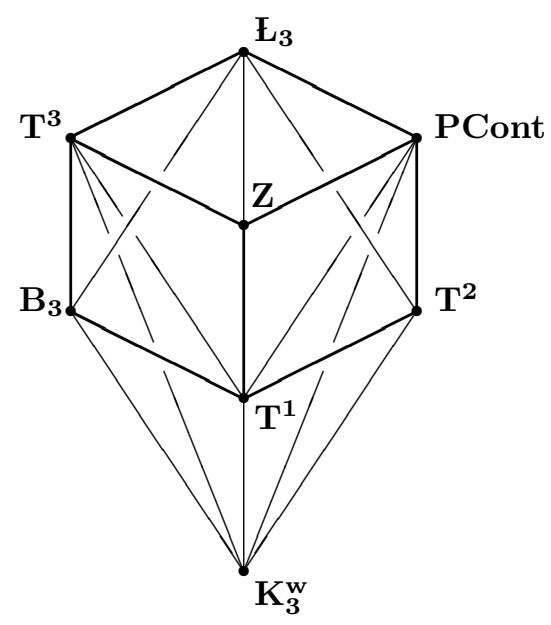

Рис. 4

Итак, логика $\mathbf{H}_{\mathbf{3}}$ отсутствует в данной классификации, поскольку не содержит ни одну из 28 естественных импликаций. Известно, что эта логика находится между $\mathbf{K}_{\mathbf{3}}^{\mathbf{w}}$ и $\mathbf{B}_{\mathbf{3}}$.

Логика Эббингауза $\mathbf{E}_{\mathbf{3}}$ также не попадает в эту классификацию, поскольку содержит импликации из двух классов $\mathbf{B}_{\mathbf{3}}$ и $\mathbf{Z}$ (см. таблицу разбиений 28 импликаций) и, что важно, заменить их на одну импликацию (импликацию какого-то одного класса) нельзя, т.е. $\mathbf{E}_{\mathbf{3}}$ не является импликативным расширением $\mathbf{K}_{\mathbf{3}}^{\mathbf{w}}$. Чтобы получить $\mathbf{E}_{\mathbf{3}}$, необходимо по крайней мере 2 раза последовательно расширить $\mathbf{K}_{\mathbf{3}}^{\mathbf{w}}$ : сначала расширить $\mathbf{K}_{\mathbf{3}}^{\mathbf{w}}$ до $\mathbf{B}_{\mathbf{3}}$, а затем посредством любой импликации из класса $\mathbf{Z}$ до $\mathbf{E}_{\mathbf{3}} .{ }^{17}$

$\mathbf{E}_{\mathbf{3}}$ находится между $\mathbf{B}_{\mathbf{3}}$ и $\mathbf{E}_{\mathbf{3}}$. Интересно, что у $\mathbf{E}_{\mathbf{3}}$ имеется некоммутативный напарник $\mathbf{T}^{\mathbf{4}}$ (точно также как у $\mathbf{B}_{\mathbf{3}}$ имеется $\mathbf{T}^{\mathbf{2}}$, а у $\mathbf{P C o n t}$ имеется $\mathbf{T}^{\mathbf{3}}$ ). Так же как $\mathbf{E}_{\mathbf{3}}$ получается за счет расширения логики $\mathbf{B}_{\mathbf{3}}$ посредством импликаций из класса $\mathbf{Z}$ (или за счет расширения логики $\mathbf{Z}$ посредством импликаций из

\footnotetext{
${ }^{16}$ Напомним, логику Холдена можно рассматривать как расширение $\mathbf{K}_{\mathbf{3}}^{\mathbf{w}}$ посредством добавления $J_{\frac{1}{2}}$-оператора.

${ }^{17}$ Аналогичным образом $\mathbf{E}_{3}$ можно получить, сначала расширив $\mathbf{K}_{3}^{\mathbf{w}}$ до $\mathbf{Z}$, а затем посредством любой импликации из класса $\mathbf{B}_{\mathbf{3}}$ до $\mathbf{E}_{\mathbf{3}}$.
} 
класса $\mathbf{B}_{\mathbf{3}}$ ), аналогичным образом логика $\mathbf{T}^{\mathbf{4}}$ получается расширением логики $\mathbf{T}^{\mathbf{2}}$ посредством добавления импликаций из класса $\mathbf{Z}$ (или за счет расширения логики $\mathbf{Z}$ посредством импликаций из класса $\mathbf{T}^{2}$ ). Так же как $\mathbf{E}_{\mathbf{3}}, \mathbf{T}^{\mathbf{4}}$ не является импликативным расширением $\mathbf{K}_{\mathbf{3}}^{\mathbf{w}}$ и непосредственно в представленную классификацию не входит.

Суммируем полученные в ходе исследования результаты и представим импликативные расширения всех трех регулярных логик $\mathbf{K}_{\mathbf{3}}, \mathbf{K}_{\mathbf{3}} \rightarrow$ и $\mathbf{K}_{\mathbf{3}}^{\mathbf{w}}$ следующим образом:

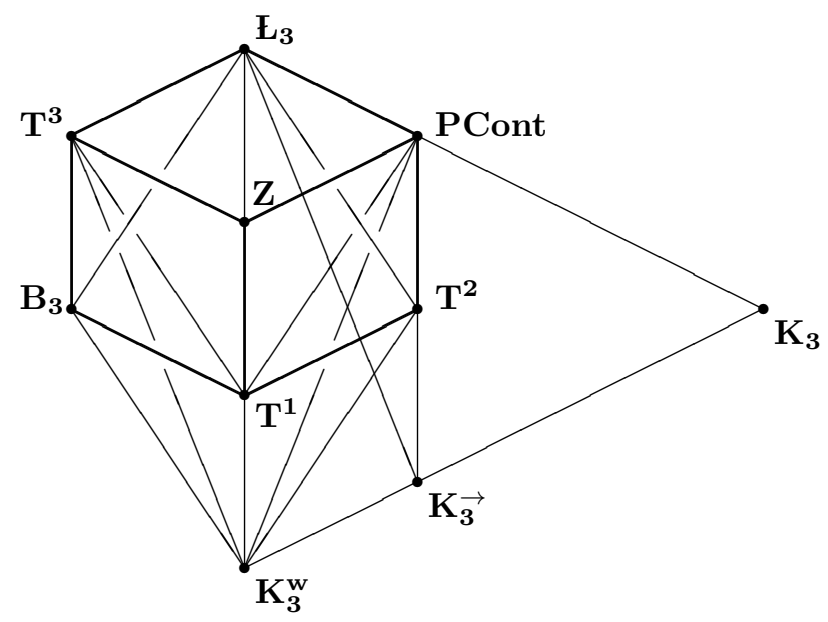

Рис. 5

Итак, в качестве импликативных расширений регулярных логик Клини выступают 7 базовых логик: $\mathbf{L}_{\mathbf{3}}, \mathbf{P C o n t}, \mathbf{B}_{\mathbf{3}}, \mathbf{Z}, \mathbf{T}^{\mathbf{3}}$, $\mathbf{T}^{\mathbf{2}}$ и $\mathbf{T}^{\mathbf{1}}$. Таким образом, установлено, что для построения логик $\mathbf{Ł}_{3}$ и PCont в качестве основания может выступать любая регулярная логика, в основе логики $\mathbf{T}^{\mathbf{2}}$ может лежать или $\mathbf{K}_{\mathbf{3}} \rightarrow$ или $\mathbf{K}_{\mathbf{3}}^{\mathbf{w}}$, в то время как логики $\mathbf{T}^{\mathbf{1}}, \mathbf{Z}, \mathbf{T}^{\mathbf{3}}, \mathbf{B}_{\mathbf{3}}$ появляются исключительно как импликативные расширения слабой логики Клини $\mathbf{K}_{\mathbf{3}}^{\mathbf{w}}$.

Необходимо отметить, что во всех представленных импликативных расширениях, имеет место стандартная теорема дедукции, поскольку в каждой из 7 систем имеется такая импликация, что $K$ и $S$ являются тавтологиями: 
$K . p \rightarrow(q \rightarrow p)$

$S .(p \rightarrow(q \rightarrow r)) \rightarrow((p \rightarrow q) \rightarrow(p \rightarrow r))$.

\section{Литература}

[1] Бочвар Д.А. Об одном трехзначном исчислении и его применении к анализу парадоксов классического расширенного функциональого исчисления // Математический сборник. 1938. Т. 4. № 2. С. 287-308.

[2] Карпенко А.C. Многозначные логики. Серия «Логика и компьютер». М.: Наука, 1997.

[3] Клини С.К. Введение в метематематику. М.: ИЛ, 1957.

[4] Комендантская Е.Ю. Функциональная взаимовыразимость регулярных логик Клини // Логические исследования. Вып. 15. 2009. С. 116-128.

[5] Розоноэр Л.И. О выявлении противоречий в формальных теориях. I // Автоматика и телемеханика. 1983. № 6. С. 113-124.

[6] Томова H.E. О расширениях логики Lisp // Шестые Смирновские чтения: материалы Междунар. науч. конф., Москва, 17-19 июня 2009 г. М.: Современные тетради. 2009. С. 104-106.

[7] Финн B.K. О предполноте класса функций, соответствующего трехзначной логике Лукасевича // Научно-техническая информация. Серия 2. 1969. № 10. C. $35-38$.

[8] Финн В.К. Аксиоматизация некоторых трехзначных исчислений высказываний и их алгебр // Философия и логика. М.: Наука, 1974. С. 398-438.

[9] Финн В.К. О критерии функциональной полноты для В3 // Исследования по формализованным языкам и неклассическим логикам. М.: Наука, 1974. С. 194199.

[10] Anderson A. R., Belnap N. D. Entailment: The Logic of Relevance and Necessity. Princeton University Press. 1975.

[11] Avron A. Natural 3-valued logics - characterization and proof theory // The Journal of Symbolic Logic. 1991. Vol. 56. № 1. P. 276-294.

[12] Batens D. Paraconsistent extensional propositional logics // Logique et Analyse. 1980. Vol. 23. № 90-91. P. 127-139.

[13] Ebbinghaus H.-G. Uber eine pradikatenlogik mit partiell definierten Pradikaten und Funktionen // Arch. Math. Logik Grundlagenforsch. 1969. Bd. 12. P. 39-53.

[14] Fitting $M$. Kleene's three valued logics and their children // Fundamenta Informaticae. 1992. Vol. 20. P. 113-131.

[15] Hatkowska K. A note on matrices for systems of nonsens-logic // Studia Logica. 1989. Vol. 48. №4. P. 461-464.

[16] Hallden S. The Logic of Nonsense, Uppsala, 1949.

[17] Jaśkowski S. Rahunek zdan dla systemov dedukcyjnych sprzecznych // Studia Societatis Scientiarum Torunensis. 1948. 1(5), Sectio A (Engish translation: propositional calculus for contradictory dedactive systems // Studia Logica. 1969. Vol. 24. P. 143-157)

[18] Eukasiewicz J. O logice trójwartosciowey // Ruch Filozoficzny. 1920. Vol. 5. 170171.(English translation: On three-valued logic // Łukasiewicz J. Selected works. PWN. Warszawa. 1970. P. 87-88.)

[19] Eukasiewicz J., Tarski A. Investigations into the sentential calculus // Łukasiewicz J. Selected Works. Amsterdam \& Warszawa: North-Holland \& PWN. 1970.

[20] Monteiro A. Construction des algèbres de Łukasiewicz trivalentes dans les algèbres de Boole monadiques, I // Mathematica Japonica. 1967. Vol. 12. P. 1-23.

[21] Rasiowa H. An Algebraic Approach to Non-classical Logics. Amsterdam: NorthHolland. 1974. 
[22] Resher N. Many-valued Logic. New York: McGraw-Hill. 1969.

[23] Sette A.M. On propositional calculus P1 // Mathematica Japonica. 1973. Vol. 16. P. $173-180$.

[24] Slupecki E., Bryll J., Prucnal T. Some remarks on the three-valued logic of J. Łukasiewicz // Studia Logica. 1967. Vol. 21. P. 45-70.

[25] Sobocinsski B. Axiomatization of a partial system of three-valued calculus of propositions // The Journal of Computin Systems. 1952. Vol. 11. №1. P. 23-55. 